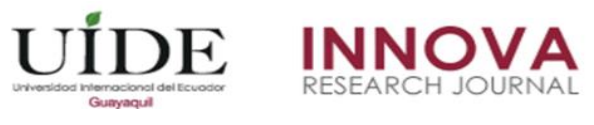

INNOVA Research Journal, ISSN 2477-9024

(Diciembre, 2018). Vol. 3, No.12 pp. 38-54

DOI: https://doi.org/10.33890/innova.v3.n12.2018.697

URL: http://revistas.uide.edu.ec/index.php/innova/index

Correo: innova@uide.edu.ec

\title{
Aceptación, rechazo y control parental asociados a características sociodemográficas de padres en Cuenca, Ecuador
}

\section{Parental acceptance, rejection and control in relation to sociodemographic characteristics of parents in Cuenca, Ecuador}

\author{
María Dolores Palacios \\ Fernando Villavicencio \\ Ruth Clavijo \\ Gardenia Conforme \\ Nube Arpi \\ Universidad de Cuenca, Ecuador \\ Catalina Mora \\ Universidad Nacional de Educación, Ecuador \\ Autora para correspondencia: maria.palaciosm@ucuenca.edu.ec; \\ gardenia.conforme88@ucuenca.edu.ec; nube.arpip@ucuenca.edu.ec \\ Fecha de recepción: 08 de junio de 2018 - Fecha de aceptación: 01 de diciembre de 2018
}

Resumen: El presente estudio de tipo descriptivo y correlacional, tiene un enfoque cuantitativo. Esta investigación se basa en la necesidad de identificar la dimensión de aceptación - rechazo parental para el ejercicio positivo de la paternidad. El objetivo fue analizar las relaciones entre las conductas de aceptación - rechazo y el control parental con la tipología familiar, la edad, el sexo y el nivel educativo de los progenitores. "Participaron 569 padres y madres de niños de primero a séptimo de educación básica de las escuelas públicas y privadas de Cuenca", y se aplicó el cuestionario de Rohner \& Khaleque Parent Parq//Control (2005). En el análisis estadístico, además de valores descriptivos se emplearon pruebas estadísticas no paramétricas. Como resultado se obtuvo que los progenitores manifiesten frecuentes expresiones de afecto y control y, escasas expresiones de indiferencia, agresión y rechazo. Se encontró también que el afecto e indiferencia no dependen del sexo, edad o nivel educativo de los padres; así mismo se evidenció que la agresión, el rechazo y el control son más frecuentes en progenitores menores a 36 años; los que cuentan con un nivel de instrucción básica registraron expresiones más frecuentes de control. Se constatan más afecto en las familias nucleares, en tanto que la agresión, indiferencia, rechazo y control son similares en todos los tipos de familias estudiadas. En conclusión, con independencia a las condiciones sociodemográficas, predominan padres y madres que manifiestan afecto y control hacia sus hijos.

Palabras clave: aceptación; rechazo; control parental; características sociodemográficas de los padres Abstract: This descriptive and correlational study has a quantitative approach. This research is based on the need to identify the acceptance dimension - parental rejection for the positive exercise of paternity. The objective of this research was to determine how the dimensions of affection, rejection, and parental control relate to family typologies, age, gender and level of education of parents. The Rohner Parent Parq/Control survey was applied to 569 parents, whose children attend public and private schools of Cuenca, from first to seventh grade, and the Rohner \& Khaleque Parent Parq/Control questionnaire (2005) was applied. In the statistical analysis, in addition to descriptive values, nonparametric statistical tests were used. The results indicated that parents from Cuenca to frequently 
display affection and control, and barely express indifference, aggression and rejection. Also, it was found that affection and indifference do not depend on gender, age or level of education of parents. In addition, the study revealed that aggression, rejection and control are more common in parents who are under 36 years old. Parents that only attended primary school tend to express more control than the rest. Furthermore, affection is mostly displayed in nuclear families, whereas aggression, indifference, rejection and control are present in all the studied families. In conclusion, regardless of sociodemographic factors, most parents manifest acceptance, affection and control towards their children.

Key words: parental acceptance; rejection and control; sociodemographic characteristics of parents

\section{Introducción}

La socialización es una de las funciones más ampliamente identificadas de la familia por sus efectos en la personalidad y ajuste del niño y adolescente. Torío, Peña \& Rodríguez (2008) señalan que "las estrategias de socialización que los padres utilizan con sus hijos tienen que ver con la aceptación-rechazo, calor-frialdad, afecto-hostilidad, proximidad-distanciamiento y con conductas de control". El estudio de los estilos de socialización parental puede ser abordado desde tres perspectivas de análisis diferentes: i) modelos teóricos basados en tipologías, donde teóricos proponen diferentes tipos de estilos educativos parentales y su incidencia en el desarrollo infantil y adolescente; ii) modelo dimensional donde se estudia el proceso de interacción de padres e hijos a través de dimensiones específicas, y, iii) el modelo contextual ecológico, que recogen las aportaciones de ambos modelos y buscan una integración. Si bien, estas perspectivas aportan niveles de análisis y resultados muy valiosos para explicar la conducta parental, en el presente estudio, se aborda el modelo dimensional de la Teoría Interpersonal de Aceptación y Rechazo (IPARTheory), de Rohner (2014) para describir las expresiones de aceptación - rechazo y control parental presentes en la interacción con los hijos, para prevenir comportamientos de riesgos y potenciar factores de protección en los padres y madres.

La extensa investigación transcultural a lo largo de medio siglo en la IPARTheory han sido corroborados en estudios realizados a nivel mundial "Un metanálisis realizado con 10943 participantes de 10 países mostró que tanto la aceptación materna como paterna percibida en la infancia correlaciona significativamente con la personalidad en niños y adolescentes" (Klaleque \& Rohner, 2002). Por otra parte, un estudio realizado en España reporta que el afecto y el control positivo, juega un papel decisivo en el ajuste psicosocial y comportamental de los hijos, sobre aspectos concretos de la personalidad como es el autoestima (Torío, Peña \& Inda, 2008).

Para Rohner (2016) los niños y niñas, en cualquier parte del mundo, "necesitan una forma específica de respuestas en forma de aceptación positiva de los padres/madres y otros cuidadores primarios". En este sentido, para Torío (2017) el afecto es una de las dimensiones que más atención ha recibido por parte de los investigadores de la socialización familiar.

En esta línea, como lo indica Rohner \& Carrasco (2014), el afecto físico, verbal, simbólico, reflejan la dimensión de calidez y aceptación de los padres o cuidadores principales. Estas conductas de aceptación parental produce: una autoestima más alta y bienestar psicológico (Cerezo, Casanova, De la Torre \& Carpio, 2011; Darling \& Steinberg, 1993; Oliva, Parra \& Sánchez-Queija, 2002; Steinberg \& Silverberg, 1986), un mejor ajuste escolar o competencia académica (Im-Bolter, Zadeh \& Ling, 2013; Mounts \& Steinberg, 1995; Steinberg, Lamborn, 
Dournbusch \& Darling, 1992), asociaciones significativas con altos niveles de competencia social y ajuste psicológico (Carillo, Ripoll-Núñez \& Cabrera, 2009; Torío et al., 2008; TurPorcar, Mestre, Samper \& Malonda, 2012), un menor consumo de sustancias tóxicas en los hijos (Barhr \& Hoffman, 2010; Becoña et al., 2013; Calafat, García, Juan, Becoña \& FernándezHermida, 2014; Parra \& Oliva, 2006), una menor presencia de problemas de conducta según refieren (Aunola, Stattin \& Nurmi, 2000; Pelegrina, García \& Casanova, 2002).

Por otra parte, diversos estudios que se reportan a continuación, refieren que cuando la "necesidad de afecto no es satisfecha, los niños/niñas tienden a ser hostiles, agresivos y emocionalmente inestables", o "cuando los hijos/hijas perciben rechazo, parecen estar más predispuestos hacia los problemas y trastornos de conducta y a involucrarse en el consumo de drogas y alcohol entre otros" (Rohner, Khaleque \& Cournoyer, 2012). La percepción de rechazo y crítica, especialmente del padre, y el poco afecto de la madre, se relaciona con los diferentes perfiles de victimización, agresión implicados en la dinámica del bullying y muestran poca confianza en otras personas (Gracia, Lila \& Musitu, 2005; León del Barco, Castaño, Polo del Río \& Fajardo-Bullón, 2015), asimismo, otro estudio reporta la relación entre el rechazo psicológico (crítica, manipulación, amenazas, retirada de afecto de los padres) con la agresividad de los hijos (Nishikawa, Sundbom \& Hägglöf, 2010). Se ha demostrado que el rechazo que ejerce la madre predice la agresividad impulsiva en los hijos e hijas adolescentes (García-Linares \& Carpio, 2015).

Con referencia a lo anterior, es conveniente destacar que las prácticas parentales coercitivas explica un 14,5\% de la varianza de la agresión reactiva o impulsiva, y el 12,4\% de la agresión premeditada, según un estudio realizado en España (Penado, 2012).

Según Rohner (2016), la aceptación versus rechazo es una dimensión que se extiende a lo largo de un continuo que va desde la aceptación hasta el rechazo. De manera que en un extremo se ubican las conductas de amor y afecto verbal, físicas o simbólicas, mientras que en el otro extremo se encontraría el "rechazo parental, que se refiere a la ausencia o retirada significativa de conductas o sentimientos de afecto y la presencia de una variedad de conductas psicológicas y físicamente dañinas". Para este autor, el rechazo parental puede adoptar tres formas: a) hostilidad y agresividad; b) indiferencia y negligencia $y, c)$ rechazo indiferenciado.

Si bien, el conjunto de estas expresiones se conjugan en una sola dimensión bipolar aceptación y rechazo parental, el autor de la IPARTheory, plantea el estudio del control parental, para explorar el grado de control que los padres producen sobre la conducta de los hijos, para lo cual, Rohner (1987) desarrolla la Escala de Control. Rohner et al. (2012) señalan que el control parental, es una dimensión igualmente continua, en un polo de la conducta parental está la permisividad y en el otro extremo el control restrictivo, entre estos dos polos se encuentra el control moderado y firme que, a veces, se combinan para formar un control flexible.

Cabe señalar, que el autor no realiza una descripción conceptual de estas formas de control, lo que podría generar confusión en la interpretación de los resultados. Asimismo, debemos señalar que las expresiones de afecto, indiferencia, agresión, rechazo y control son prácticas parentales que deben ser analizadas en el contexto étnico y cultural donde se manifiesten (Rohner \& Carrasco, 2014). Rodrigo \& Palacios (1998) postulan que las prácticas 
parentales "se relacionan con una serie de factores que se dividen en tres grupos: el primero relacionado con el niño, un segundo relativo a los padres; $y$, un tercer factor relacionado con la situación o contexto en que se presenta la interacción”.

Los autores de la investigación, privilegian las características de los padres y madres, al considerar que en la crianza subyacen las expresiones de aceptación - rechazo y control parental y están determinadas por el sexo de los padres, la experiencia previa como hijos y como padres, las características de personalidad, el nivel educativo, las ideas acerca del proceso evolutivo y las expectativas de logro que tienen puestas los padres en sus hijos, por ejemplo como lo señala Rodrigo \& Palacios (1998) algunos padres tienen como meta que sus hijos sean autónomos y responsables a diferencia de otros, cuyas metas son que sus hijos sean conformistas y sumisos; para el primer caso los padres utilizan el diálogo para establecer normas y en el segundo caso, las estrategias de crianza serán ordenar, imponer y castigar.

En cuanto al sexo de los padres, Putnick et al (2012); Rohner \& Carrasco (2014), reportaron que "la aceptación de los padres (versus madres) tuvo una relación más fuerte con el ajuste psicológico de los niños que la aceptación de las madres". Por otra parte, en un estudio en España, Linares \& Fernández (2015), reportaron el rechazo y la disciplina rígida de la madre y la disciplina indulgente del padre. Las diferencias de los progenitores en cuanto a la disciplina parental, como una forma de control parental, indican que "las madres de España y Estados Unidos aplicaron con mayor frecuencia la mayoría de los métodos disciplinarios que los padres" (Gámez-Guadix \& Almendros, 2015). Así mismo, Rodríguez, Del Barrio \& Carrasco (2009) encontraron un mayor grado de control por parte de las madres.

De la misma manera, una investigación con familias indígenas mexicanas, reveló que las hijas perciben ser más controladas por sus madres que los hijos (Esteinou, 2015). Por otra parte estudios desarrollados en los últimos años en el contexto español y latinoamericano, parecen demostrar que el control flexible utilizado por padres y madres, es tan efectivo e incluso más que el control firme en el desarrollo de los adolescentes (Calafat et al., 2014; Fuentes, Alarcón, García \& García, 2015; García \& Gracia, 2010; Villalobos, Cruz \& Sánchez, 2004; Martínez, García \& Yubero, 2007). Asimismo, en el Ecuador, Schvaneveldt (2014) una investigación con 1400 padres y madres, encontró que las madres interactúan con los hijos usando estrategias de inducción positiva, y padres y madres monitorizan las actividades de los hijos. Atendiendo a estos resultados, los autores de la investigación señalan que las manifestaciones de aceptación, prácticas de control, y monitorización están relacionadas con el sexo de los padres.

Entre los factores asociados a los progenitores, el más consistente relacionado a los estilos educativos ha sido la clase social o nivel socioeconómico, entendida como la combinación del nivel educativo, profesión, nivel de ingresos y calidad de la vivienda (Ramírez, 2005). El nivel de estudios es el que más ayuda a diferenciar unos progenitores de otros en relación a los estilos educativos. En este sentido, estudios reportan que un menor nivel educativo de los padres está asociado con prácticas más severas, mientras que niveles educativos más elevados se relacionan con prácticas más inductivas (Gámez-Guadix \& Almendros, 2015). Estos resultados difieren de un estudio en Uruguay donde los padres y madres con nivel de educación primaria aparecieron significativamente más inductivos que los padres con educación terciariauniversitaria (Capano, González \& Massonnier, 2016). 
En cuanto a la tipología familiar, Martínez-Monteagudo, Estebez \& Cándido (2013) indican que en la actualidad aunque la familia nuclear sigue predominando en buena parte de las sociedades, la proporción ha disminuido notablemente, así, hoy en día son mucho más comunes las familias reconstituidas resultantes de divorcios o nuevas nupcias, las familias monoparentales compuestas por un solo progenitor, también las familias extendidas conformadas por personas de varias generaciones; este tipo de familia responde a una cultura colectivista como la China, comunidades de etnia gitana y familias Latinoamericanas.

En esta dirección, no se han encontrado diferencias significativas en cuanto al estilo educativo en familias de varias configuraciones, ni en los estilos parentales (Capano et al., 2016; Olivia \& Arranz, 2011).

En otro orden de ideas, Ramírez (2005) indica que "entre las circunstancias que influyen sobre las ideas de los progenitores y sus prácticas de crianza, están las experiencias de socialización, las limitaciones o posibilidades relacionadas con los hijos/hijas, las ideas específicas respecto a sus capacidades", las experiencias previas como progenitores, la forma en que los padres/madres recuerdan su propia educación cuando fueron menores; así como el bienestar económico (Carter \& Middlemiss, 1992) y la personalidad de los padres (Dix, 1991), como también Hidalgo (1988) sugiere que la edad de los padres, podría explicar que a mayor edad las personas serían emocionalmente más maduras para afrontar el rol de padres.

Teniendo en cuenta los resultados de los estudios mencionados, sostenemos que las características de los padres se relacionan con la aceptación - rechazo y control en la interacción con los hijos; claro está, sin desconocer el enfoque bidireccional y el ecológico - contextual, modelos actuales de la socialización familiar.

Después de las consideraciones anteriores, los autores proponen como objetivos de esta investigación: determinar la frecuencia del afecto, indiferencia, agresión, rechazo y control parental, como expresiones que integran la dimensión de aceptación-rechazo que los progenitores cuencanos manifiestan en la interrelación con sus hijos, y analizar las expresiones de afecto, calidez, indiferencia, agresión, rechazo y control parental relacionadas con el perfil sociodemográfico como: edad, sexo, instrucción y tipología familiar de los padres y madres cuencanos. Finalmente, esperamos que la descripción de las dimensiones de aceptación- rechazo y control parental, se consideren como una referencia para encontrar la forma de relacionarse, y educar a los hijos.

\section{Materiales y método}

Esta investigación de enfoque cuantitativo, corresponde a un nivel descriptivocorrelacional. Como variables predictivas se consideraron las características de los padres: edad, sexo, nivel de educación y la tipología familiar, en tanto que las expresiones de aceptación y rechazo que utilizan los padres y madres cuencanos con niños escolares de 5 a 12 años de edad constituyeron la variable criterio.

\section{Muestra de la población}


Se trabajó con 569 padres y madres de niños de entre 5 y 12 años, estudiantes de 10 escuelas fiscales y 10 particulares de la ciudad de Cuenca seleccionadas por método probabilístico polietápico: por conglomerados - estratificado. Los conglomerados constituyeron los centros educativos que ofertan Educación General Básica (EGB), el criterio para la estratificación fue el tipo de sostenimiento de la institución. El tamaño de la muestra se calculó con un nivel de confianza del $95 \%(\mathrm{z}=1.96)$ y un error muestral del $4 \%$.

Los participantes: $49.6 \%$ padres y $50.4 \%$ madres, en su mayoría casados $(82.4 \%)$, registraron una media de edad de 36.55 años (DT= 6.99 años), con un mínimo de 22 años y un máximo de 71 años. El 46.7\% de ellos señaló haber alcanzado instrucción superior, el 75.4\% realiza una actividad por la que reciben remuneración y reportó una media de 8.36 horas diarias dedicadas al trabajo ( $\mathrm{DE}=1.98$ horas), con un mínimo de 2 y un máximo de 16 horas.

Los participantes responden a cuatro tipos de familias: nucleares $(68 \%)$, extendidas $(18.9 \%)$, monoparentales $(11.7 \%)$ y reconstituidas $(1.4 \%)$. Ver Tabla 1.

Tabla 1

Características demográficas de la muestra $(\mathrm{n}=569)$

\begin{tabular}{llllll}
\hline Variables & & $\%$ & Variables & \\
\hline \multirow{2}{*}{ Sexo } & Hombres & 49.6 & Actividad & Con & 75.4 \\
& Mujeres & 50.4 & remunerada & Sin & 24.2 \\
& Ninguno & 0.7 & & $<8$ horas & 11.6 \\
Nivel de & Básica & 20.6 & Jornada de & $8-12$ horas & 62.2 \\
instrucción & Bachillerato & 31.5 & trabajo & $>12$ horas & 1.1 \\
& Superior & 46.7 & & NC & 25.1 \\
& NC & 0.5 & & & \\
& Nucleares & 68 & & Casado & 82.4 \\
\multirow{5}{*}{ Tipo de familia } & Monoparentales maternas & 10.6 & Situación & Separado & 8.1 \\
& Monoparentales paternas & 1.2 & conyugal & Divorciado & 4.7 \\
& Reconstituidas & 1.4 & & Soltero & 2.5 \\
& Total & 100 & & Viudo & 0.5 \\
\hline
\end{tabular}

\section{Instrumentos}

La información se recolectó mediante una encuesta autoaplicada, se empleó un cuestionario para padres y uno para madres, estructurado en dos secciones: datos sociodemográficos, tipología familiar y la escala Parent Parq/ Control, versión corta (Ronher \& Klaleque 2005).

La escala Parent Parq/Control incluye 29 Ítems, 5 corresponden a expresiones de control y 24 a la dimensión aceptación rechazo, integrada por cuatro tipos de expresiones: 8 Ítems para cariño /afecto, 6 para hostilidad/agresión, 6 para indiferenciada/negligencia y 4 Ítems para rechazo indiferenciado. Las respuestas responde a una escala tipo Likert abreviada de 4 puntos: siempre $=4$, muchas veces $=3$, pocas veces $=2$, nunca $=1$.

En cada expresión, puntuaciones elevadas indican frecuentes manifestaciones de aceptación, indiferencia (3 Ítems inversos), agresión, rechazo (1 Ítem inverso) y control (1 Ítem 
inverso). Para determinar la autopercepción de los progenitores acerca de la conducta de aceptación y rechazo con sus hijos, se invierte la puntuación de la escala cariño/afecto (mide frialdad) y se suma a los puntajes registrados en las subescalas: indiferencia, agresión y rechazo; en este caso, puntuaciones bajas corresponden a frecuentes manifestaciones de afecto y puntuaciones altas corresponden a frecuentes manifestaciones de rechazo. La escala aceptaciónrechazo (24 Ítems) representa un continuo que va de 24 a 96 puntos, puntajes cercanos a 24 implican frecuentes expresiones de afecto y escasas expresiones de rechazo. Para esta escala se registró una alta consistencia interna con un alfa de Cronbach $=.86$ la dirigida a los padres y .84 la dirigida a las madres; en tanto que la escala control (5 Ítems) reportó un alfa de Cronbach de .592 en el caso de los padres y de .597 en el caso de las madres.

\section{Recolección de los datos}

Para el acceso a las unidades muestrales, se gestionó la autorización de la Coordinación Zonal 6 de Educación del cantón Cuenca, se coordinó la aplicación con los directores de las instituciones educativas, con ayuda de los docentes de cada grado y por intermedio de los niños de primero a séptimo de educación básica (primaria) se envió a los padres y madres, una carta informativa, la hoja de consentimiento informado y el cuestionario, en sobre cerrado. Previo a la construcción de la matriz de datos, se realizó el control de calidad y se clasificaron los cuestionarios como: completos/válidos e incompletos/no válidos.

\section{Análisis de datos}

La descripción de las cuatro expresiones de la dimensión aceptación-rechazo y del control parental, se realizó mediante: frecuencias porcentuales, gráficos de distribución de frecuencias, medidas de tendencia central y variabilidad. En la generalización de resultados se usaron intervalos de confianza y para el análisis bivariado pruebas no paramétricas: Kruskal Wallis y UMann Withney, ya que tras la aplicación de la prueba de Kolmogorov-Smirnov se determinó que las distribuciones de las variables de estudio no cumplieron con la condición de normalidad (p < .05). El procesamiento de datos se realizó con el programa estadístico SPSS versión 22, las decisiones se tomaron con una significancia del $5 \%(\mathrm{p}=.05)$.

\section{Resultados}

La dimensión afecto registró una media de 29.26 ( $\mathrm{DE}=3.13)$, valor cercano al puntaje máximo de la subescala, lo cual implica frecuentes expresiones de afecto. Ver Figura 1a. Por su parte las dimensiones: indiferencia $(\mathrm{M}=9.28 ; \mathrm{DE}=2.49)$, agresión $(8.54 ; \mathrm{DE}=1.95)$ y rechazo $(\mathrm{M}=4.89 ; \mathrm{DE}=1.07)$, reportaron medias con valores cercanos al valor mínimo de cada una de dichas subescalas, lo cual representa escasas manifestaciones de: indiferencia, agresión y rechazo parental. Ver figura $1 \mathrm{~b}, 1 \mathrm{c}, 1 \mathrm{~d}$. 


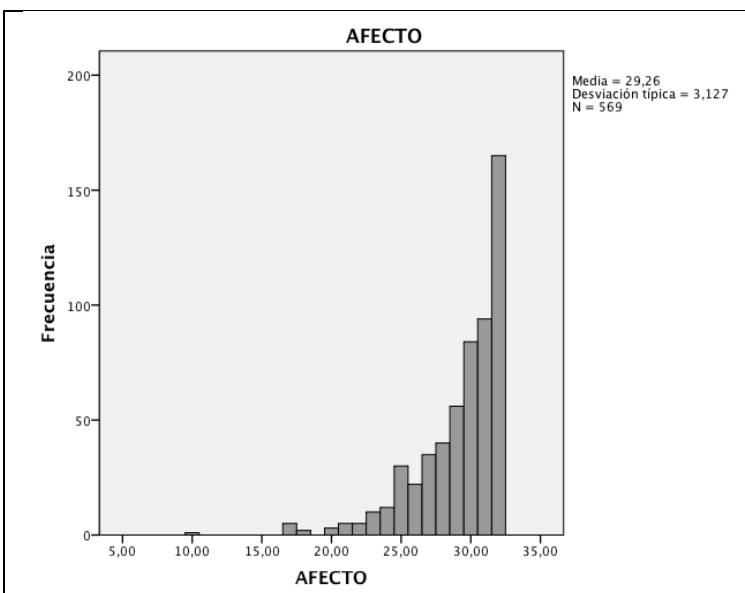

Figura 1a. Puntajes afecto

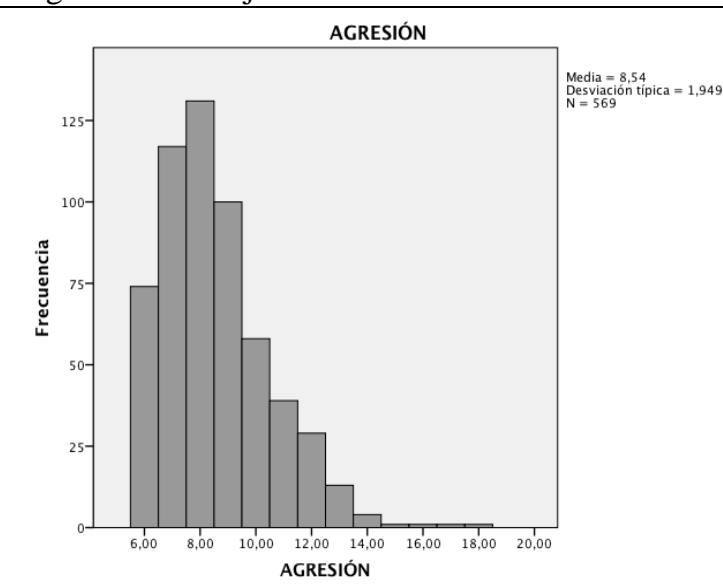

Figura 1c. Puntajes agresión

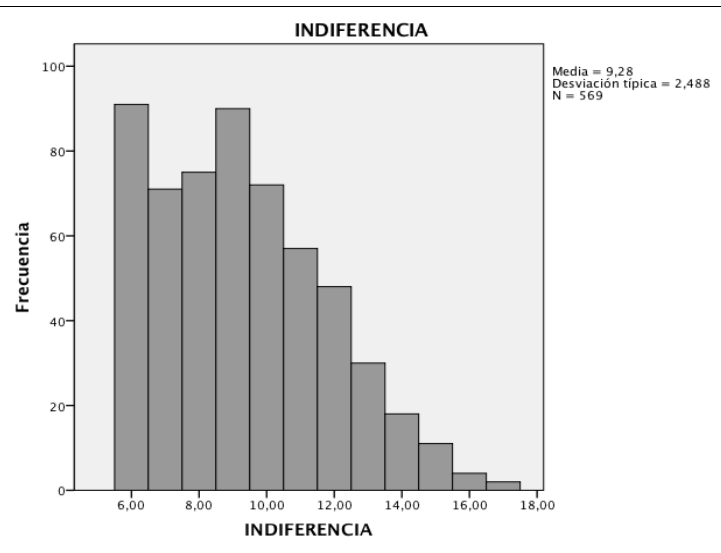

Figura 1b. Puntajes indiferencia

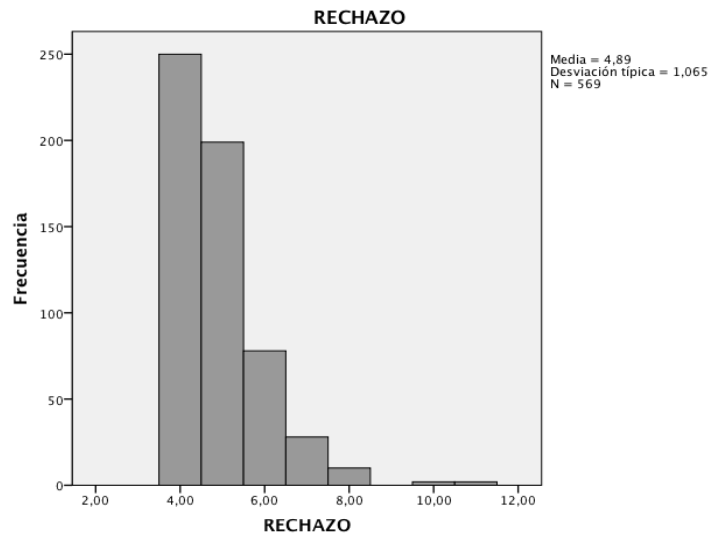

Figura 1d. Puntajes rechazo

\section{Figura 1. Distribución de puntajes de la dimensión aceptación- rechazo}

La variable aceptación-rechazo registró una media de 33.42 (DE=6.6), un IC 95\% [32.88, 33.97] valores que reflejan una tendencia hacia habituales manifestaciones de afecto y escasas manifestaciones de rechazo de padres y madres cuencanos hacia sus hijos. Ver Figura 2a. Adicionalmente se encontró una tendencia hacia constantes muestras de control parental $(\mathrm{M}=$ $15.75 ; 2.39)$. Ver Figura $2 b$. 


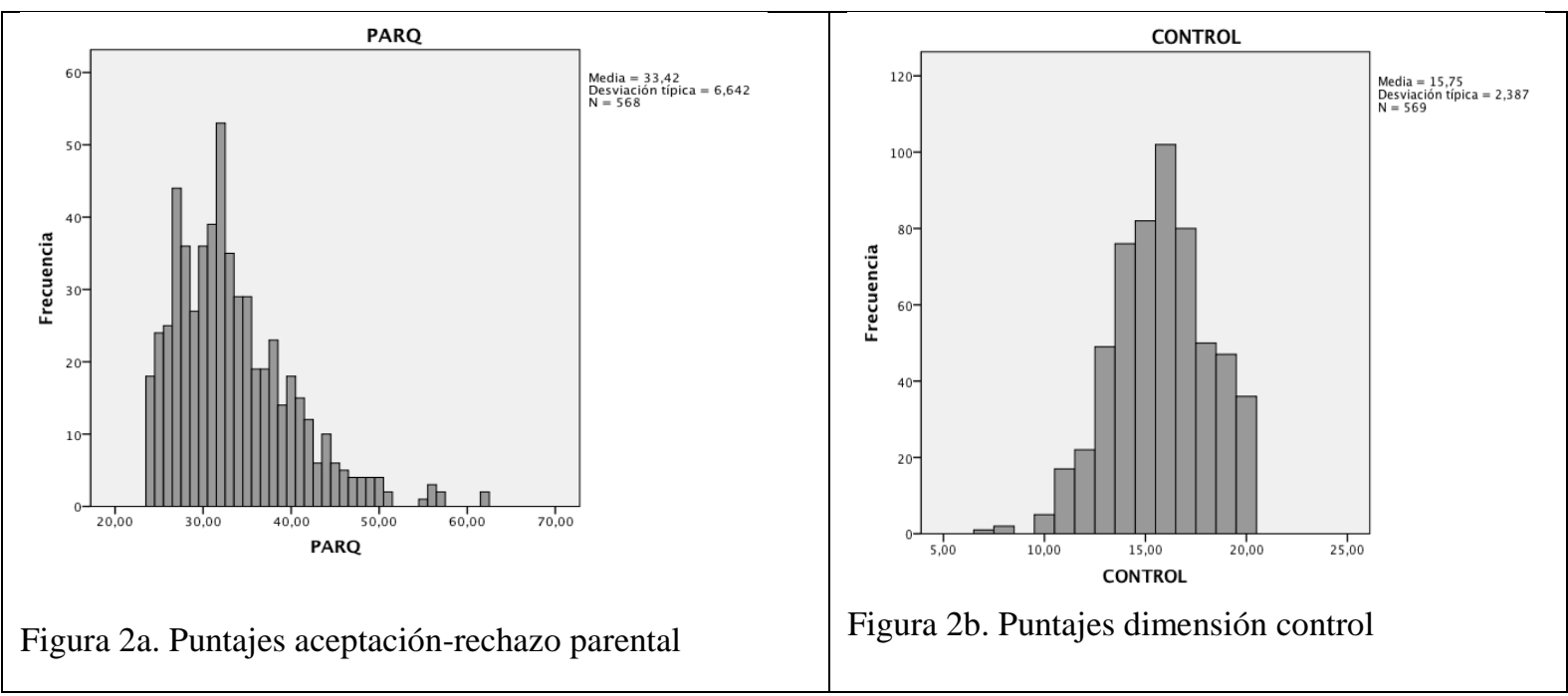

Figura 2. Distribución de puntajes de la dimensión aceptación, rechazo y control

La Tabla 2 detalla los porcentajes de participantes en función de la frecuencia de las expresiones de: afecto, indiferencia, agresión, rechazo y control hacia sus hijos. Así el 94.6\% de progenitores reportaron frecuentes (muchas veces y siempre) manifestaciones de afecto hacia sus hijos y un $69.8 \%$ frecuentes expresiones de control. En general se reportaron escasas expresiones de: indiferencia, agresión y rechazo; un $58.3 \%$ de padres señaló que pocas veces expresan indiferencia a sus hijos; de igual forma, el $43.2 \%$ respondió que pocas veces expresa agresión y un $21.1 \%$ que pocas veces rechaza a sus hijos.

Tabla 2.

Frecuencia de las expresiones de aceptación-rechazo parental

\begin{tabular}{lllll}
\hline Dimensiones & Nunca & Pocas veces & Muchas veces & Siempre \\
\hline Afecto & $0.2 \%$ & $5.3 \%$ & $17.4 \%$ & $77.2 \%$ \\
Indiferencia & $41.7 \%$ & $58.3 \%$ & ----- & ---- \\
Agresión & $56.6 \%$ & $43.2 \%$ & $0.2 \%$ & ----- \\
Rechazo & $78.9 \%$ & $21.1 \%$ & ----- & ----- \\
Control & $0.2 \%$ & $30.1 \%$ & $46.4 \%$ & $23.4 \%$ \\
\hline
\end{tabular}

La Tabla 3, incluye los Ítems que registraron las puntuaciones más significativas en las expresiones de afecto, indiferencia, agresión, rechazo y control parental, en ella se observa que a pesar que los padres reportaron frecuentes expresiones de afecto, el porcentaje que siempre dice cosas buenas de sus hijos y se preocupa sobre lo que piensa y le gusta al hijo para hablar sobre eso es de aproximadamente el $50 \%$.

Aunque las expresiones de rechazo son escasas, en lo que a indiferencia parental se refiere, cerca del 5\% de progenitores respondió que frecuentemente (siempre o muchas veces) está demasiado ocupado para contestar las preguntas de su hijo o que olvida cosas importantes de su hijo que debería recordar. De igual forma, respecto a las expresiones de agresión aproximadamente el $10 \%$ de progenitores mencionó que con frecuencia pega a su hijo cuando se lo merece o atemoriza o amenaza a su hijo cuando hace algo mal. Finalmente, menos del $2 \%$ de progenitores reportaron frecuentes expresiones de rechazo. 
Respecto al control, el $63.8 \%$ de progenitores señaló que siempre se preocupan de que su hijo sepa exactamente lo que puede o no puede hacer, el $62.2 \%$ siempre le dice a su hijo cómo debe portarse, en tanto que, un $90.7 \%$ menciona que nunca o pocas veces deja que su hijo haga lo que quiera hacer.

\section{Tabla 3}

\section{Expresiones de afecto-rechazo y control parental}

\begin{tabular}{|c|c|c|c|c|c|}
\hline \multirow[t]{2}{*}{ Dimensiones } & \multirow[t]{2}{*}{ Ítems (indicadores) } & Siempre & $\begin{array}{l}\text { Muchas } \\
\text { veces }\end{array}$ & $\begin{array}{l}\text { Pocas } \\
\text { veces }\end{array}$ & Nunca \\
\hline & & $\%$ & $\%$ & $\%$ & $\%$ \\
\hline \multirow{4}{*}{ Afecto } & Hago saber a mi hijo que le quiero & 86.1 & 10.7 & 2.5 & 0.7 \\
\hline & $\begin{array}{l}\text { Estoy realmente interesada en los asuntos de mi } \\
\text { hijo }\end{array}$ & 78.6 & 18.3 & 2.8 & 0.4 \\
\hline & Yo digo cosas buenas de mi hijo & 57.8 & 35.7 & 6.5 & 0 \\
\hline & $\begin{array}{l}\text { Me preocupo sobre lo que piensa y le gusta a mi } \\
\text { hijo para hablar sobre eso }\end{array}$ & 51.7 & 35.1 & 11.2 & 1.9 \\
\hline \multirow{2}{*}{ Indiferencia } & $\begin{array}{l}\text { Estoy demasiado ocupada para contestar las } \\
\text { preguntas de mi hijo }\end{array}$ & 0.9 & 4.6 & 48.9 & 45.7 \\
\hline & $\begin{array}{l}\text { Me olvido cosas importantes de mi hijo que } \\
\text { debería recordar }\end{array}$ & 2.1 & 2.3 & 43.4 & 52.2 \\
\hline \multirow[b]{2}{*}{ Agresión } & Pego a mi hijo cuando se lo merece & 2.3 & 6.9 & 61.9 & 29 \\
\hline & $\begin{array}{l}\text { Atemorizo o amenazo a mi hijo cuando hace } \\
\text { algo mal }\end{array}$ & 1.6 & 8.3 & 49 & 40.9 \\
\hline \multirow{4}{*}{ Rechazo } & La presencia de mi hijo me molesta & 0.4 & 0 & 9 & 90.7 \\
\hline & Me gusta como es mi hijo & 78.6 & 20 & 1.2 & 0.2 \\
\hline & $\begin{array}{l}\text { Cuando mi hijo se porta mal le niego muestras } \\
\text { de cariño }\end{array}$ & 0.4 & 3 & 40.9 & 55.7 \\
\hline & Permito saber a mi hijo que no es querido & 1.9 & 0 & 1.9 & 96.1 \\
\hline \multirow[t]{2}{*}{ Control } & $\begin{array}{l}\text { Me preocupo de que mi hijo sepa exactamente } \\
\text { lo que puede o no puede hacer }\end{array}$ & 63.8 & 28.6 & 7.2 & 0.4 \\
\hline & Yo le digo a mi hijo cómo debe portarse & 62.2 & 26.2 & 10.9 & 0.7 \\
\hline
\end{tabular}

Nota: Los valores destacados en negro corresponden a los puntajes que los autores consideran más representativos. *Ítem inverso

Se encontró que la frecuencia de las expresiones de afecto es diferente según el tipo de familia, siendo más frecuentes en las familias nucleares, seguida por la monoparental materna; en tanto la frecuencia de las expresiones de: agresión, indiferencia, rechazo y control es similar en todos los tipos de familia. Ver Tabla 4.

Tabla 4.

Expresiones de aceptación-rechazo parental según el tipo de familia

\begin{tabular}{|c|c|c|c|c|c|c|}
\hline \multirow{2}{*}{ Expresiones } & Nuclear & Reconstituida & Extendida & Monoparental & \multirow{2}{*}{$\begin{array}{c}\text { Kruskal } \\
\text { Wallis }\end{array}$} & \multirow{2}{*}{$\mathrm{p}$} \\
\hline & $\mathrm{M}(\mathrm{DT})$ & $\mathrm{M}(\mathrm{DT})$ & $\mathrm{M}(\mathrm{DT})$ & $\mathrm{M}(\mathrm{DT})$ & & \\
\hline Afecto & $29.48(2.87)$ & $28.33(1,21)$ & 28.66 (3.35) & $28.91(4.32)$ & 8.288 & $.04^{*}$ \\
\hline Agresión & 8.57 (1.93) & $10(1.41)$ & $8.35(1.97)$ & $8.57(2.04)$ & 6.073 & 0.108 \\
\hline Indiferencia & $9.2(2.41)$ & $9.67(2.54)$ & $9.65(2.73)$ & $9.09(2.49)$ & 2.962 & 0.397 \\
\hline Rechazo & $4.88(1.07)$ & $4.67(0.52)$ & $4.93(1.13)$ & $4.89(0,91)$ & 0.393 & 0.942 \\
\hline Control & $15.8(2.31)$ & $16.33(1.63)$ & $15.73(2.51)$ & $15.28(2.75)$ & 1.534 & 0.674 \\
\hline
\end{tabular}

Nota: Los valores destacados en negro corresponden a los puntajes que los autores consideran más representativos. * Diferencia significativa a nivel de .05 
Se encontró que la frecuencia de las manifestaciones de afecto e indiferencia parental es similar entre las madres y los padres y entre los progenitores de las diferentes edades. Ver Tabla 5. Por su parte, las expresiones de agresión son más frecuentes en las madres que en los padres y en los progenitores menores a 36 años (media de la muestra); en cuanto a la frecuencia de las expresiones de rechazo, las madres manifiestan rechazo a sus hijos con mayor frecuencia que los padres. Se reportó además, mayor control por parte de las madres que de los padres y de los progenitores menores a 36 años respecto a los mayores a 36 años. Ver Tabla 5.

\section{Tabla 5}

Aceptación-rechazo y control según el sexo y la edad de los progenitores

\begin{tabular}{|c|c|c|c|c|c|c|}
\hline \multirow{3}{*}{ Expresiones } & \multicolumn{2}{|l|}{ Sexo } & \multirow{3}{*}{$\mathrm{p}$ valor } & \multicolumn{2}{|l|}{ Edad } & \multirow{3}{*}{$\mathrm{p}$ valor } \\
\hline & padres & madres & & $\square 36$ años & $\square 36$ años & \\
\hline & $\mathrm{M}(\mathrm{DT})$ & $\mathrm{M}(\mathrm{DT})$ & & $\mathrm{M}(\mathrm{DT})$ & $\mathrm{M}(\mathrm{DT})$ & \\
\hline Afecto & $29.1(3.2)$ & $29.4(3.1)$ & .242 & $29.16(3.24)$ & $29.41(2.93)$ & .677 \\
\hline Agresión & $8.2(1.7)$ & $8.87(2.1)$ & $.000 * *$ & $8.7(2)$ & 8.37 (1.89) & $.037 *$ \\
\hline Indiferencia & $9.43(2.6)$ & $9.14(2.4)$ & .247 & $9.24(2.54)$ & $9.33(2.42)$ & .483 \\
\hline Rechazo & $4.73(0.9)$ & $5.05(1.2)$ & $.001 * *$ & $4.94(1.14)$ & $4.84(.99)$ & .47 \\
\hline Control & $15.5(2.4)$ & $15.9(2.4)$ & $.015^{*}$ & $15.96(2.38)$ & $15.54(2.39)$ & $.034 *$ \\
\hline
\end{tabular}

* Diferencia significativa a nivel de .05

** Diferencia significativa a nivel de .01

Se encontró que la frecuencia de las manifestaciones de afecto e indiferencia parental son similares entre los progenitores de los distintos niveles educativos; la frecuencia de las expresiones de rechazo registra mayor frecuencia en los progenitores con instrucción superior. Finalmente, se reportó mayor control de los progenitores con instrucción básica, respecto a los progenitores con bachillerato o nivel superior. Ver Tabla 6

Tabla 6

Expresiones de aceptación-rechazo parental y nivel educativo de los progenitores

\begin{tabular}{llllll}
\hline \multirow{2}{*}{ Expresiones } & Básica & Bachillerato & Superior & \multirow{2}{*}{ Kruskal Wallis } & p \\
\cline { 2 - 4 } & $\mathrm{M}$ & $\mathrm{M}$ & $\mathrm{M}$ & & 0.269 \\
Afecto & $29.14(3.5)$ & $29.35(3.38)$ & $29.23(2.77)$ & 2.625 & 0.113 \\
agresión & $8.29(1.78)$ & $8.5(1.98)$ & $8.71(1.98)$ & 4.366 & 0.32 \\
indiferencia & $9.2(2.55)$ & $9.14(2.49)$ & $9.43(2.47)$ & 2.278 & $0.004^{* *}$ \\
Rechazo & $4.65(0.86)$ & $4.85(1.06)$ & $5.03(1.14)$ & 11.051 & $0.000^{* *}$ \\
Control & $17.07(2.29)$ & $16.13(2.2)$ & $14.88(2.22)$ & 75.812 & \\
\hline
\end{tabular}

* Diferencia significativa a nivel de $\mathbf{. 0 5}$

** Diferencia significativa a nivel de .01

\section{Discusión}

El presente estudio examinó las expresiones de aceptación-rechazo y control parental y relación con la tipología familiar, el sexo, la edad y el nivel de educación de los padres y madres cuencanos. El primer objetivo fue determinar la frecuencia de las expresiones de la dimensión aceptación-rechazo (afecto, indiferencia, agresión y rechazo) y control parental, desde la percepción de los progenitores. Los resultados evidencian que los progenitores de niños/niñas entre 5 y 12 años de edad, reportan altas puntuaciones en las expresiones de afecto, así el 86.1\% manifiesta que "siempre hace saber a sus hijos que los quieren", también registran altos puntajes 
en las expresiones de control, el $63.8 \%$ de progenitores responde "siempre me preocupo de que mi hijo sepa exactamente lo que puede y no puede hacer". La frecuentes expresiones de afecto y control, coexisten con escasas expresiones de indiferencia, agresión y rechazo; conducta que denota aceptación parental.

Estos hallazgos mostraron similitud en cuanto a las altas puntuaciones de afecto y control encontrado por Schvaneveldt (2014) y se corrobora con las altas puntuaciones de afecto reportadas por Putnick et al. (2012). En este sentido Khaleque \& Rohner (2002), estimaron que alrededor del $75 \%$ de padres mundialmente son cálidos y amorosos con sus hijos, y el restante $25 \%$ son caracterizados por tener un leve rechazo.

Es necesario destacar que, el $2 \%$ de padres/madres reportaron frecuentes expresiones de rechazo, esto implica que existiría un porcentaje similar de población infantil de Cuenca que podría percibir rechazo de sus padres. A pesar que este estudio no valoró las consecuencias del rechazo en los niños, no puede descartarse lo que reportan otros estudios, que los hijos que perciben rechazo, parecen estar predispuestos a conductas asociadas a la victimización, agresión, a trastornos de conducta y al consumo de drogas y alcohol entre otros (Gracia et al., 2005; León del Barco et al., 2015; Rohner et al., 2012).

Con respecto al segundo objetivo, determinar la relación del perfil sociodemográfico de los progenitores con las expresiones de aceptación - rechazo parental, se encontró que las manifestaciones de control, agresión y rechazo por parte de las madres hacia sus hijos, tienden a ser mayores que las de los padres. En otras palabras, las madres se muestran más controladoras, agresivas y rechazadoras que los padres. Investigaciones han reportado resultados de las prácticas parentales, coherentes con lo reportado por los hijos (Rodríguez, Del Barrio \& Carrasco, 2009) es así, que un estudio reporta que los hijos perciben a las madres mexicanas como más controladoras que los padres (Esteinou, 2015). Asimismo, García-Linares \& Carpio (2015) en una investigación realizada en España, indican que el rechazo y la disciplina rígida, como una expresión de control es ejercida por la madre frente a la falta de disciplina y la indulgencia del padre. Las diferencias de los padres en cuanto a la disciplina parental indican que las madres emplean más agresión psicológica, tareas como castigo, distracción, explicación verbal, recompensas y mayor control que los padres (Gámez-Guadix \& Almendros, 2015).

De una manera general, se puede indicar que aunque los padres y madres están igualmente capacitados para proporcionar cuidados a sus hijos, en el contexto ecuatoriano convertirse en madre o padre no significa lo mismo, pues las madres continúan siendo las principales responsables de la disciplina. Esto es consistente con los resultados encontrados en estudios que indican que los padres están menos implicados en la vida diaria de sus hijos que las madres (Rodríguez et al., 2009; Tur-Porcar et al., 2012).

En cuanto a la edad, los resultados reportan que los padres/madres cuencanos menores de 36 años registraron expresiones de agresión y control con mayor frecuencia que los mayores de 36 años. Estos datos podrían sugerir estar relacionados con el hecho que las personas de mayor edad serían emocionalmente más maduras y estarían más preparadas para afrontar el nuevo rol de padre o madre (Hidalgo, 1998). 
Las manifestaciones del control parental, también se relaciona con el nivel educativo de los padres, Montoya-Castilla, Prado-Gascó, Villanueva-Badenes, \& González-Barrón (2016) encontraron que un mayor nivel educativo se asoció con el empleo de más estrategias disciplinarias inductivas (explicación verbal) y castigos no severos (tareas como castigo y comportamientos como compensación). Además, el mayor nivel educativo estuvo relacionado con menor uso de castigo físico entre las madres.

Respecto a la relación entre el nivel educativo de los padres y madres, en el presente estudio, aproximadamente el $78 \%$ de padres registraron educación superior y bachillerato, lo que podría explicar que los puntajes en las dimensiones de afecto y control estén por encima de la media de la escala de medida y que los valores en las expresiones de agresión, rechazo e indiferencia se ubiquen por debajo de la media de la escala de la IPARTheory, si bien estos resultados corroboran lo reportado por Gámez- Guadix \& Almendros (2015) que:

Un menor nivel educativo está asociado con el empleo de estrategias más punitivas, mientras que niveles educativos más elevados se relacionan con estrategias disciplinarias inductivas; sin embargo, poca es la información que revele que la relación entre nivel educativo y la dimensión de aceptación-rechazo es consistente, pues es posible que el reducido nivel educativo de los padres esté relacionado con los factores estresores del contexto como la pobreza, el desempleo, los ambientes de riesgo o la escasa información sobre conductas de crianza apropiadas que repercuten negativamente sobre las conductas parentales.

A pesar de ello, el sexo de los progenitores parece ser una variable determinante en las expresiones de agresión, rechazo y control pues aunque los niveles educativos de madres y padres son similares, las madres registraron mayores puntajes en estos tipos de expresiones. Estas conductas, podrían reflejar que en las culturas colectivistas como la ecuatoriana, por un lado las madres asumen la crianza de los hijos, y por el otro ejercen más control y disciplina como una forma natural de educar a los hijos.

Se encontró que la frecuencia de las expresiones de afecto es diferente según el tipo de familia, siendo más frecuentes en las familias nucleares, seguida por la monoparental materna; en tanto la frecuencia de las expresiones de: agresión, indiferencia, rechazo y control es similar en todos los tipos de familia. Respecto a los hallazgos de la tipología familiar, las expresiones de afecto son frecuentes en las familias nucleares, seguida por la monoparental materna, este resultado, evidencia una relación entre la tipología familiar y las expresiones de afecto, siendo coincidente con los resultados presentados por (Capano et al., 2016; Olivia \& Arranz, 2011), en cuanto a relación entre tipología familiar y prácticas de crianza.

En conclusión, los resultados ponen de manifiesto que padres y madres de Cuenca, Ecuador se autoevalúan como afectuosos, esta conducta se ubica en el extremo calidez del continuo de aceptación y rechazo propuesto por la IPARTheory de Rohner; paralelamente se perciben como controladores, esta conducta podría reflejar que los progenitores de las culturas colectivistas, como la ecuatoriana, tienden a ejercer más control y disciplina sobre los hijos/hijas, como una forma de fomentar las relaciones familiares, y en relación con el sexo, la edad, el nivel de educación de los progenitores y la tipología familiar, están asociadas a las variables aceptación, rechazo y control parental. 
En cuanto a las limitaciones del estudio, debemos indicar que la deseabilidad social pudo haber constituido una limitación de la investigación. Es posible que los progenitores hayan realizado una autoevaluación positiva de las conductas de afecto, cuidado, hacia sus hijos/hijas y hayan evitado las expresiones de rechazo, pues es conocido que la crianza parental es sensible a la cultura de los participantes.

Se sugiere para posteriores investigaciones, conocer tanto la percepción que tienen los hijos de la aceptación - rechazo y control de sus progenitores o figuras cuidadoras, como las características de los hijos, para explorar el ajuste psicosocial de la personalidad en los hijos como efecto de la percepción de aceptación y rechazo parental. Como una segunda limitación de este estudio se destaca que si bien los padres y madres se identifican como controladores, no se han caracterizado los tipos de control porque la escala del ParentParq/Control, solamente permite constatar la dimensión de aceptación - rechazo y control parental.

Finalmente, de los resultados de la investigación se deducen que, aun cuando un alto porcentaje de progenitores se perciben como afectuosos con sus hijos, también existe un grupo reducido que se percibe como agresor, por lo que resulta necesario resaltar la necesidad de trabajar en programas de educación parental positiva para que padres y madres profundicen en la comprensión, por un lado, de la importancia del afecto, apoyo; y por otro, de los efectos negativos del rechazo y la agresión en el desarrollo psicosocial de los hijos e hijas, y que las expresiones de aceptación - rechazo y control parental, no son procesos mecánicos, sino que están sujetos a la interacción de las características de los progenitores y el proceso evolutivo de los hijos y de las hijas.

\section{Bibliografía}

Aunola, K., Stattin, H. \& Nurmi, J. (2000). The role of parenting styles in children's problem behavior. Child Development, 76 (6), 1144-1159. Doi: 0009-3920/2005/7606-0002

Bahr, S.J. \& Hoffman, J.P. (2010). Parenting style, religiosity, peers, and adolescent heavy drinking. Journal of Studies on Alcohol and Drugs, 71(4), 539-543.doi: dx.doi.org/10.15288/jsad.2010.71.539

Becoña, E., Martínez, U., Calafat, A., Juan M., Fernández, J.R. \& Secades, R. (2013). Parental styles and drug use: A review. Drugs: Education, Prevention and Policy, 19(1), 1-10. Doi: $10.3109 / 09687637.2011 .631060$

Calafat, A., García, F., Juan, M., Becoña, E. \& Fernández-Hermida, J.R. (2014). Which parenting styles is more protective against adolescent substance use? Evidence within the European context. Drug and Alcohol Dependence, 138(1), 185-192. Doi: dx.doi.org/10.1016/j.drugalcdep.2014.02.705.

Capano A., González, M. \& Massonnier, N. (2016). Estilos relacionales parentales: estudio con adolescentes y sus padres. Revista de Psicología (PUCP), 34(2), 413-444. doi:dx.doi.org/10.18800/psico.201602.008 
Carillo, S., Ripoll-Núñez, K. \& Cabrera, V. (2009). Relaciones familiares, calidad de vida y competencia social en adolescentes y jóvenes colombianos. SUMMA Psychological UST, 6(2), 3-18.

Carter, B. D. \& Middlemiss, W. A. (1992). The socialization of instrumental competence in families in the United States. Parent-child socialization in diverse cultures, 5(1), 107-120.

Cerezo, M., Casanova, P., De la Torre, M. \& Carpio, M. (2011). Estilos educativos paternos y estrategias de aprendizaje en alumnos de educación secundaria. European Journal of Education and Psychology, 4(1), 51-61. Doi: 10.1989/ejep.v4i1.76

Darling, N. \& Steinberg, L. (1993) Parenting style as context: an integrative model. Psychological Bulletin, 113(3), 487-796.

Dix, T. (1991). The affective organization of parenting: Adaptative and maladaptive processes. Psychological Bulletin, 110, 3-25. Doi: dx.doi.org/10.1037/0033-2909.110.1.3

Esteinou, R. (2015). Autonomía Adolescente y Apoyo y Control Parental en Familias Indígenas Mexicanas. Revista Latinoamericana de Ciencias Sociales, 13(1), 749-766. doi:10.11600/1692715x.13214230114.

Fuentes, M., Alarcón, A., García, F., \& Gracia, E. (2015). Consumo de alcohol, tabaco, cannabis y otras drogas en la adolescencia: efectos de la familia y peligro del barrio. Anales de Psicología, 31(1), 1000-1007. Doi: 10.6018/analesps.31.3.183491

García, F., \& Gracia, E. (2010). ¿Qué estilo de socialización parental es idóneo en España? Un estudio con niños y adolescentes de 10 a 14 años. Infancia y Aprendizaje, 33(1), 365-384.

Gámez-Guadix, M., \& Almendros, C. (2015). Parental discipline in Spain and in the United States: differences by country, parent-child gender and education level. Infancia y Aprendizaje, 38(3), 569-599. Doi: dx.doi.org/10.1080/02103702.2015.1054665

García-Linares, M. \& Carpio, M. (2015). Las prácticas educativas paternas y la agresividad premeditada e impulsiva de los hijos adolescentes. Psicología Conductual, 23(1), 161-179.

Gracia, E., Lila, M. \& Musitu, G. (2005). Rechazo parental y ajuste psicológico y social de los hijos. Salud Mental, 28(2), 73-81.

Hidalgo, M. (1998). Transición a la maternidad y la paternidad. En Rodrigo y Palacios (Eds), Familia y Desarrollo Humano (pp.161-180). España: Alianza Editorial.

Im-Bolter, N., Zadeh, Z. \& Ling, D. (2013). Early parenting beliefs and academic achievement: The mediating role of language. Early Child Development and Care, 183, 1811-1826.

Khaleque, A. \& Rohner, R. P. (2002). Perceived parental acceptance-rejection and psychological adjustment: A meta-analysis of cross cultural and intracultural studies. Journal of Marriage and the Family, 64(1), 54 -64. doi:10.1111/j.1741-3737.2002.00054.x 
León Del Barco, B., Castaño, F., Polo Del Río, M. \& Fajardo-Bullón, F. (2015). Aceptación-rechazo parental y perfiles de victimización y agresión en situaciones de bullying. Anales de Psicología, 31(2), 600-606. Doi: dx.doi.org/10.6018/analesps.31.2.156391

Linares, M., \& Fernández, M. (2015). Las prácticas educativas paternas y la agresividad premeditada e impulsiva de los hijos adolescentes. Psicología Conductual, 23(1), 161.

Martínez-Monteagudo, M., Estebez, E. \& Cándido, I., (2013). Diversidad familiar y ajuste psicosoial en la sociedad actual. Revista Psicologia.com.17 (6).

Martínez, I., García, J.F. \& Yubero, S. (2007). Parenting styles and adolescents 'self-esteem in Brazil. Psychological Reports, 100(1), 731-745.

Montoya-Castilla, I., Prado-Gascó, V., Villanueva-Badenes, L., and González-Barrón, R. (2016). Adaptación en la infancia: influencia del estilo parental y del estado de ánimo. Action Psicol. 13:2. doi: 10.5944/ap.13.2.17807

Mounts, N.S. \& Steinberg, L. (1995). An ecological analysis of peer influence on adolescent grade point average and drug use. Developmental Psychology, 31, 915-922

Nishikawa, S., Sundbom, E. \& Hägglöf, B. (2010). Influence of perceived parental rearing on adolescent self-concept and internalizing and externalizing problems in Japan. Journal of Child and Family Studies, 19(1), 57-66.

Oliva, A., Parra, A. \& Sánchez. (2002). Relaciones con padres e iguales como predictoras del ajuste emocional y conductual durante la adolescencia. Apuntes de psicología, 20 (2), 225-242.

Oliva, A. \& Arranz, E. (Coords.) (2011). Nuevas familias y bienestar infantil. España: Secretariado de Publicaciones de la Universidad de Sevilla y Servicio editorial de la Universidad del País Vasco.

Parra, A. \& Oliva, A. (2006). Un análisis longitudinal sobre las dimensiones relevantes del estilo parental durante la adolescencia. Infancia y Aprendizaje, 29(4), 453-470.

Pelegrina, S., García, M. \& Casanova, P. (2002). Los estilos educativos de los padres y la competencia académica de los adolescentes. Infancia y Aprendizaje, 25(2), 147-168. Doi: dx.doi.org/10.1174/021037002317417796

Penado, M. (2012). Agresividad reactiva y proactiva en adolescentes: efecto de los factores individuales y socio-contextuales. Tesis doctoral no publicada. Universidad Complutense de Madrid, España.

Putnick, D., Bornstein, M., Lansford, E., Chang, L., Deater-Deckard, K., Di Giunta, L. \& Pastorelli, C. (2012). Agreement in mother and father acceptance-rejection, warmth, and hostility/rejection/neglect of children across nine countries. Cross-Cultural Research, 46(3), 191-223. Doi: 10.1177/1069397112440931

Ramírez, M.A. (2005). Padres y desarrollo de los hijos. Prácticas de crianza. Estudios Pedagógicos, 31(2), 167-177. Doi: dx.doi.org/10.4067/S0718-07052005000200011 
Rodríguez, M., Del Barrio, V. \& Carrasco, M. (2009). Consistencia interparental y su relación con la agresión y la sintomatología depresiva en niños y adolescentes. Revista de Psicopatología y Psicología Clínica, 14(1), 51-60.

Rodrigo, M., \& Palacios, J. (1988). La familia como contexto y la familia en contexto. En Rodrigo y Palacios (Eds), Familia y Desarrollo Humano (pp. 25-44). España: Editorial Alianza.

Rohner, R. P. y Khaleque, A. (2005). Personality assessment

Questionnaire (PAQ): Test manual. En R. P. Rohner y A. Khaleque (Eds.), Handbook for the study of parental acceptance and rejection, 4th ed. (pp. 187-226). Storrs, CT: Rohner Research Publications.

Rohner, R., Khaleque, A. \& Cournoyer, D. (2012). Introduction to parental acceptance-rejection theory, Methods, Evidence, and implications. Journal of Family Theory \& Review, 2(1), 73 87.

Rohner, R. \& Carrasco, M. Á. (2014). Teoría de la Aceptación-Rechazo Interpersonal (IPARTheory): Bases Conceptuales, Método y Evidencia Empírica. Acción Psicológica, 11(2), 9-26. Doi: dx.doi.org/10.5944/ap.11.2.14172

Rohner, R. P. (2016). Introduction to Interpersonal Acceptance-Rejection Theory (IPARTheory) and Evidence. Online Readings in Psychology and Culture, 6(1), 4. Doi: dx.doi.org/10.9707/ 23070919.1055

Schvaneveldt, P. L. (2014). Parenting in Ecuador: Behaviors that promote social competence. In H. Selin (Eds.), Parenting Across Cultures (pp. 323-334). Netherlands: Springer. Doi: 10.1007/978-94-007-7503-9_24

Steinberg, L. \& Silverberg, S. (1986). The visissitudes of autonomy in early adolescence. Child Development, 57(1), 841-851. Doi: 10.2307/1130361

Steinberg, L., Lamborn, S., Dornbusch, S. \& Darling, N. (1992). Impact of parenting practices on adolescent achievement: Authoritative parenting, school involvement, and encouragement to succeed. Child Development, 63(1), 1266-1281. Doi: 10.2307/1131532

Torío, S. (2017). ¿Cómo Educar? ¿Lo estamos haciendo bien? Contribuyendo al actual debate de la literatura acerca del estilo educativo parental óptimo. Pedagogía Social. Revista Interuniversitaria, 29(1), 9-18. Doi: 10. SE7179/PSRI_2016.29.00

Torio, S., Peña, J.V. \& Rodríguez, M.C. (2008). Estilos educativos parentales, Revisión bibliográfica y reformulación teórica. Teoría de la Educación, 20(1), 151-178.

Tur-Porcar, A., Mestre, V., Samper, P. \& Malonda, E. (2012). Crianza y agresividad de los menores: ¿es diferente la influencia del padre y de la madre? Psicothema, 24(2), 284-288.

Villalobos, J., Cruz, A. \& Sánchez, P. (2004). Estilos parentales y desarrollo psicosocial en estudiantes de Bachillerato. Revista Mexicana de Psicología, 21(1), 119-129. 